\title{
Analyse théorique-expérimentale de l'écoulement réel dans les pompes assoradiales
}

\author{
Theoretical-experimental analysis \\ of real flows in assoradial pumps
}

E. Carnevale*, S. Giusti**, G. Angelo*

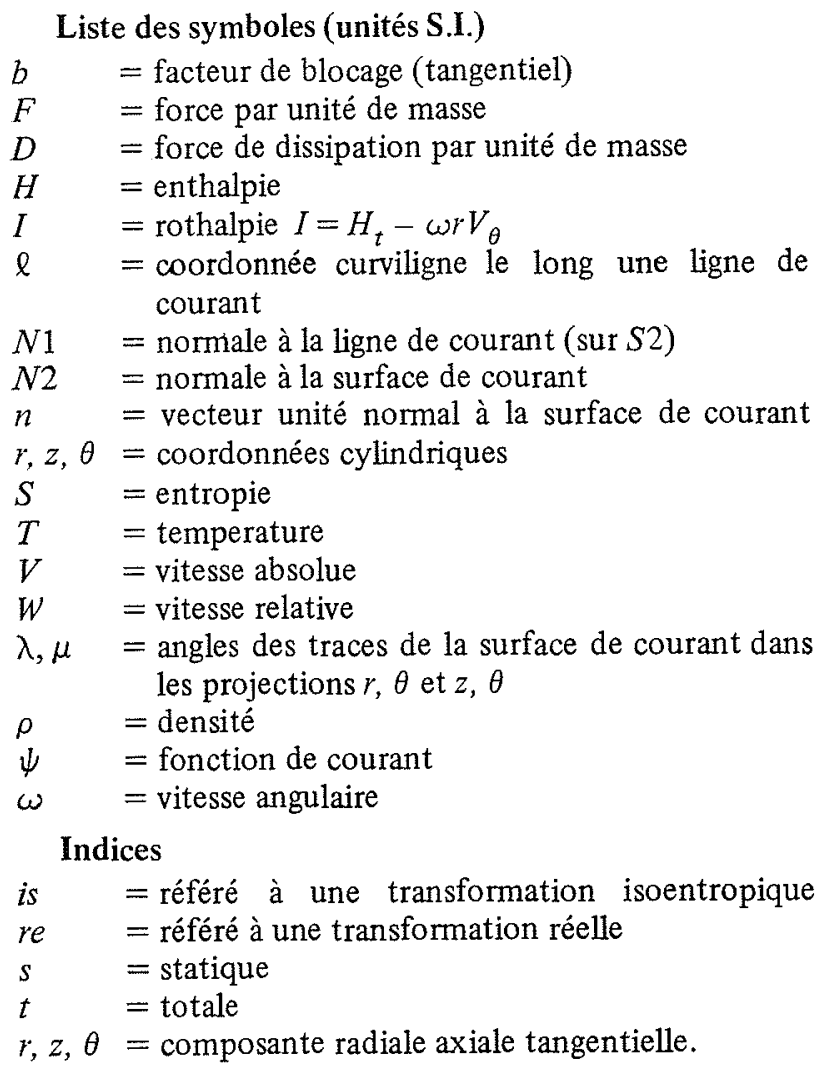

* Professeur à l'Université de Florence, Istituto di Energetica Via di S. Marta, 3 - 50139 Firenze - Italie Tel. 055-472001. ** Rechercheur à l'Université de Florence, Istituto di Energetica Via di S. Marta, 3 - 50139 Firenze - Italie Tel. 055-472001. *** Ingénieur, Riva Calzoni S.p.A. Via Stendhal, $34-20144$ Milano - Italie Tel. 02-479151.

\section{Introduction}

Le modèle numérique présenté ici est basé sur la théorie proposée par C. Bosman et $\mathrm{H}$. Marsh [1] pour l'étude de l'écoulement stationnaire d'un fluide incompressible dans les roues des turbomachines.

Une caractéristique intéressante de cette méthode est la possibilité de tenir compte des pertes grâce à un modèle compatible de dissipation. On étudie l'écoulement le long d'une surface moyenne interaubes (Surface $S 2$ suivant la théorie générale de $W_{\mathcal{L}}$ ) en présence d'un champ de forces de frottement $(\vec{D})$ qui agissent localement et tangentiellement aux lignes de courant et en opposition à la vitesse relative (Fig. 1).

L'imposition d'une surface d'écoulement foumit une équation du genre $f(r, \theta, z)=0$ qui peut remplacer une des équations du mouvement. Dans le détail on peut définir l'écoulement irréversible adiabatique relatif en utilisant les équations suivantes :

- équation de continuité $\nabla(\rho \vec{W})=0$

- équation du mouvement en direction tangentielle à la surface d'écoulement et normale à la ligne de courant relative :

$$
\overrightarrow{N 1} \cdot \vec{w}(\nabla \times \vec{V})=\overrightarrow{N 1} \cdot(\nabla I-T \nabla S)
$$

- équation du changement d'entropie le long des lignes de courant

- équation de la surface d'écoulement $f(r, \theta, z)=0(3)$

- équation de l'énergie $\frac{d I}{d t}=0$

(le long une ligne de courant $I(\psi)=$ const.)

- équation $\rho=$ constante puisque le régime est incompressible.

Ces équations sont suffisantes pour le calcul sur la surface moyenne interaubes $(S 2)$; les autres deux équations du mouvement sont automatiquement verifiées et on peut les utiliser pour le calcul des forces de frottement et des forces sur les aubes. 


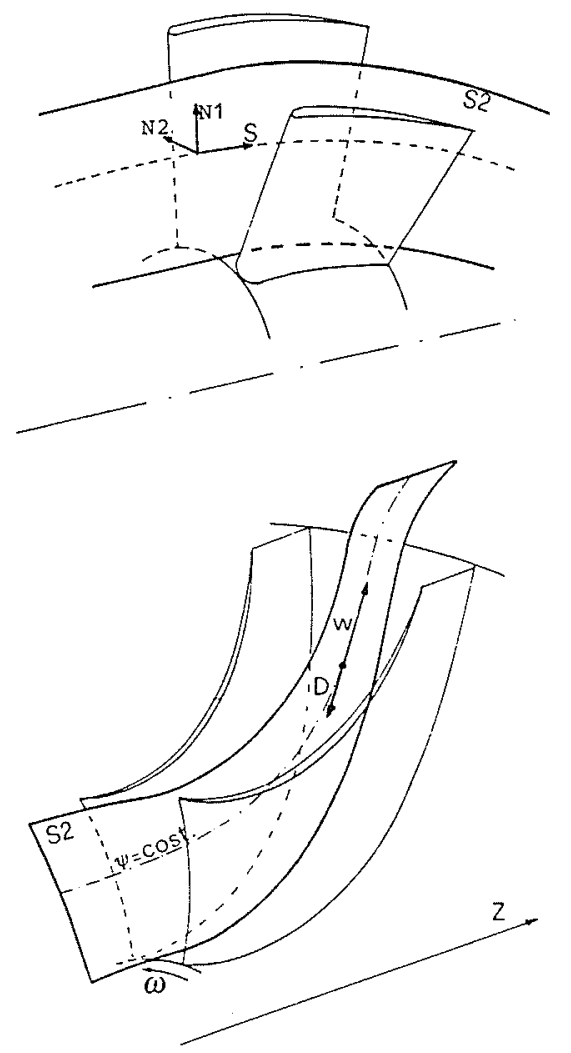

Figure 1 - Surface d'écoulement et force de frottement.

\section{Equation finale d'écoulement}

Pour l'étude de l'écoulement sur la surface moyenne $S 2$ définie par l'équation (3), il convient d'utiliser les dérivées partielles $(\bar{\theta})$ le long de la surface $S 2$; pour une fonction quelconque $g$, on a :

$$
\begin{aligned}
& \frac{\bar{\partial} g}{\partial r}=\frac{\partial g}{\partial r}-\frac{n_{r}}{r \cdot r_{\theta}} \cdot \frac{\partial g}{\partial \theta} \\
& \frac{\bar{\partial} g}{\partial z}=\frac{\partial g}{\partial z}-\frac{n_{z}}{r \cdot n_{\theta}} \cdot \frac{\partial g}{\partial \theta}
\end{aligned}
$$

En outre il est commode de définir la géométrie locale par les angles $\lambda$ et $\mu$ (Fig. 2) :

$$
\tan \lambda=\frac{n_{r}}{n_{\theta}} \quad \tan \mu=\frac{n_{z}}{n_{\theta}}
$$

et la fonction de courant $\psi$ donnée par les relations :

$$
\frac{\bar{\partial} \psi}{\partial r}=\rho b r V_{z} \quad \frac{\bar{\partial} \psi}{\partial z}=-\rho b r V_{r}
$$

En tenant compte de toutes les relations précédentes, l'équation (2) prend la forme :

$$
\frac{\bar{\partial}^{2} \psi}{\partial r^{2}}+\frac{\bar{\partial}^{2} \psi}{\partial z^{2}}=q_{I}+q_{E}
$$

où $q_{I}$ et $q_{E}$ sont données par

$$
\begin{aligned}
q_{I}=V_{z} & \frac{\bar{\partial}}{\partial r}(\rho b r)-V_{r} \frac{\bar{\partial}}{\partial z}(\rho b r)+(\rho b r)^{2} \frac{d I}{d \psi} \\
+\rho b \cdot & {\left[\operatorname{tg} \mu \cdot \frac{\bar{\partial}}{\partial r}\left(r V_{\theta}\right)-\operatorname{tg} \lambda \cdot \frac{\bar{\partial}}{\partial z}\left(r V_{\theta}\right)\right] } \\
q_{E}=-\rho & \frac{b r T}{W^{2}} \\
\cdot & {\left[\frac{\partial S}{\partial r}\left(V_{z}-W_{\theta} \operatorname{tg} \mu\right)-\frac{\partial S}{\partial z}\left(V_{r}-W_{\theta} \operatorname{tg} \lambda\right)\right] }
\end{aligned}
$$

Le terme $q_{E}$ donnant l'accroissement d'entropie le long la surface d'écoulement, peut être calculé en utilisant la méthode proposée par les auteurs [2].

Brièvement, on peut définir un coefficient de perte $\eta$, fonction de la valeur locale de $\psi$; par :

$$
\eta=\eta(\psi)=\frac{d P_{t, r e}}{d P_{t, i s}}
$$

et arriver, pour une ligne de courant quelconque, à exprimer l'accroissement locale d'entropie avec la relation :

$$
\int_{0}^{\ell} T \frac{\partial S}{\partial \ell} \cdot d \ell=[1-\eta(\psi)] \int_{0}^{\ell} \frac{\partial H_{t, i s}}{\partial \ell} d \ell
$$

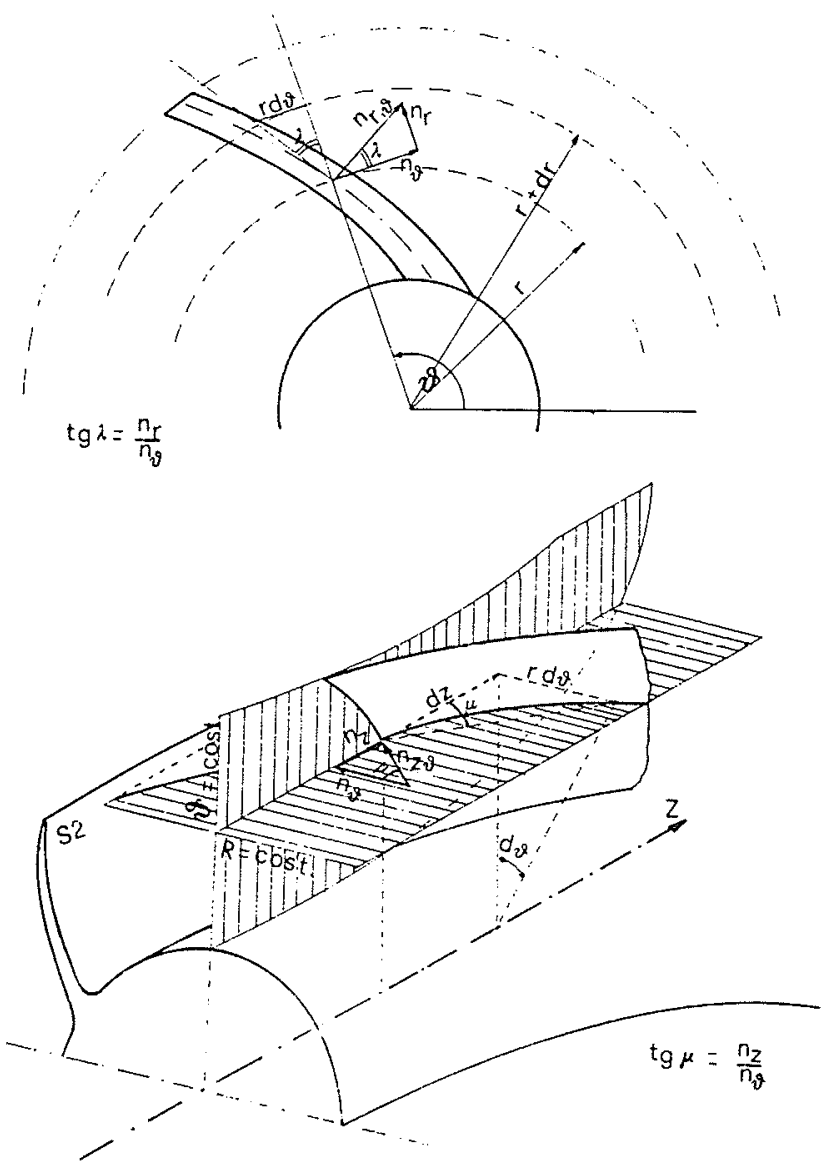

Figure $2-$ Angles $\lambda$ et $\mu$. 
La distribution $\eta(\psi)$ du coefficient de perte peut être déterminée directement sur la base de mesures de la pression totale à la sortie de la roue, ou sur la base des expériences accumulées par la même famille de pompes. Le mérite de la méthode est de permettre le calcul du champ d'entropie dans la roue, en utilisant les distributions de pression totale hors de l'aubage.

\section{Solution numérique}

Une procédure préliminaire doit être développée pour définir la géométrie de la surface $S 2$. Les données de départ sont les coordonnées et les épaisseurs d'aubes sur quelque filet de la surface moyenne d'aubes. En général, il est suffisant d'avoir les données sur les filets de pied (hub) et de tête (shroud) de la roue, sur le filet moyen et sur les deux filets intermédiaires.

Pour chaque filet, on calcule le facteur de glissement à la sortie de la roue en utilisant l'expression proposée par Wiesner [3]. Pour la répartition du coefficient de glissement le long du filet on formule l'hypothèse de distribution suivant l'expression :

$$
1-\sigma=\left(1-\sigma_{s}\right)\left[\frac{r-r_{i}}{r_{s}-r_{i}}\right]^{3}
$$

où $\sigma$ est le coefficient de glissement, les indices $\mathrm{i}, \mathrm{e}, \mathrm{s}$, sont référés à l'entrée et à la sortie de la roue.

La répartition de l'effet de glissement sur tous les filets d'aubes donnés permet de définir la géométrie de la surface $S 2$. Toute la procédure préliminaire est basée sur l'utilisation de "splines" paramétrique du troisième degré pour l'interpolation tridimensionnelle des courbes sur les surfaces géométriques et sur la surface $S 2$.

Pour la résolution du problème de l'écoulement sous forme discrète on utilise la méthode des éléments finis (éléments isoparamétriques quadrilatères à huit points nodaux).

La recherche de la solution de l'équation finale (7) est effectuée par la méthode des résidus pesés (Galerkin), en assumant pour les fonctions-poids les fonctions-types elles-mêmes.

La séquence des procédures de calcul développées dans le modèle numérique est représentée dans l'organigramme de la figure 3 .

\section{Calcul de l'écoulement dans une roue assora- diale}

Le modèle numérique a été utilisé pour le calcul de l'écoulement dans une roue assoradiale avec vitesse spécifique $N_{q}=80$ (Nombre typique en unité S.I., $k=1,54$ ).

La figure 4 fournit le tracé du canal méridien. On a effectué le calcul pour le débit d'adaptation $\left(Q_{N}\right)$, pour un débit inférieur $\left(0,75 . Q_{N}\right)$ et un débit supérieur $\left(1,2 Q_{N}\right)$.

Les calculs préliminaires, ici présentés, ont été effectués sans indications expérimentales spécifiques; pour les conditions à la sortie de la roue, on a calculé le coefficient de glissement suivant la méthode proposé

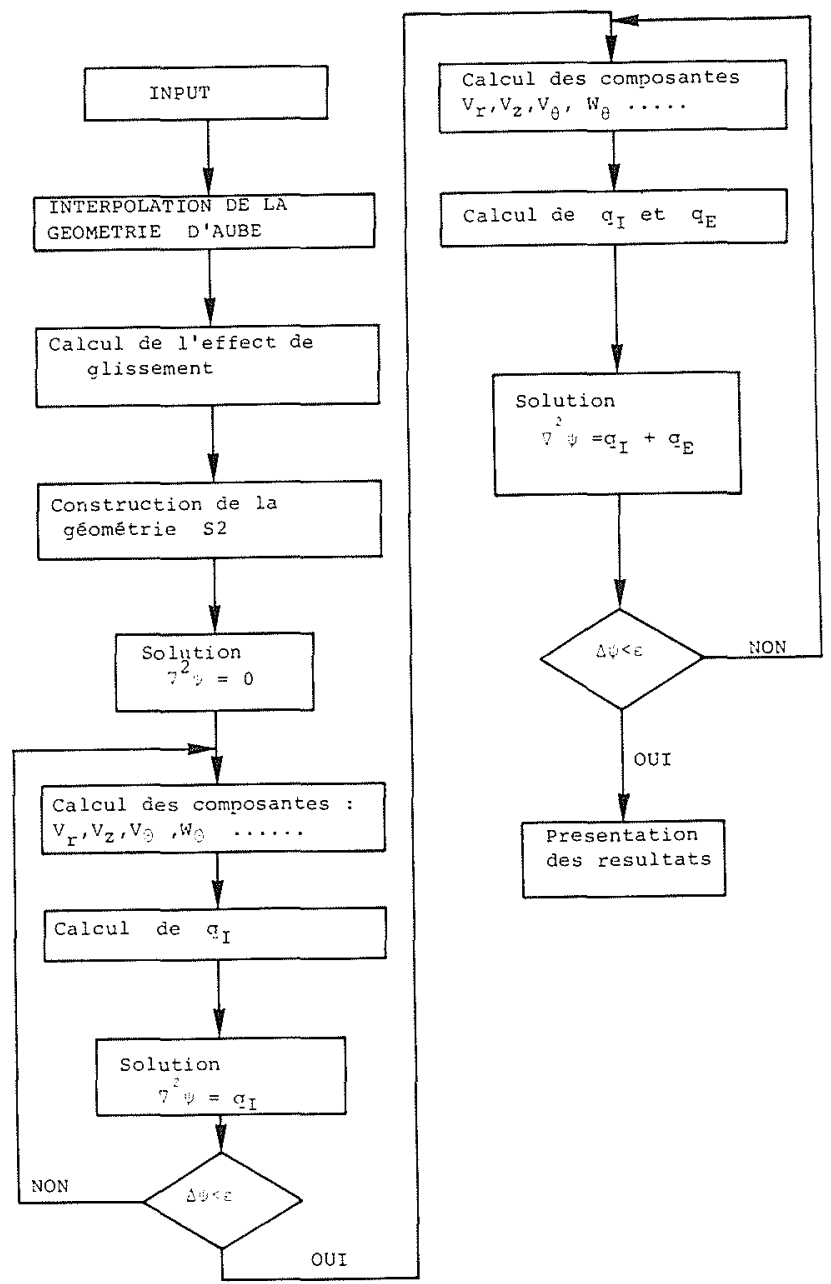

Figure 3 - Organigramme des calculs.

par Wiesner [3] et on a défini la surface $S 2$ selon la procédure exposée au paragraphe 3 .

En outre, on a prolongé cette surface avant l'entrée de la roue jusqu'à pouvoir considérer une vitesse pratiquement axiale. Par la suite, on a fait les relèvements expérimentaux sur la pompe avec deux sondes Pitot à 5 trous insérés à l'entrée et à la sortie de la roue.

Les figures 5, 6, 7, 8 illustrent la comparaison entre valeurs mesurées et calculées pour les angles relatifs à la sortie de la roue et pour les composantes de la vitesse.

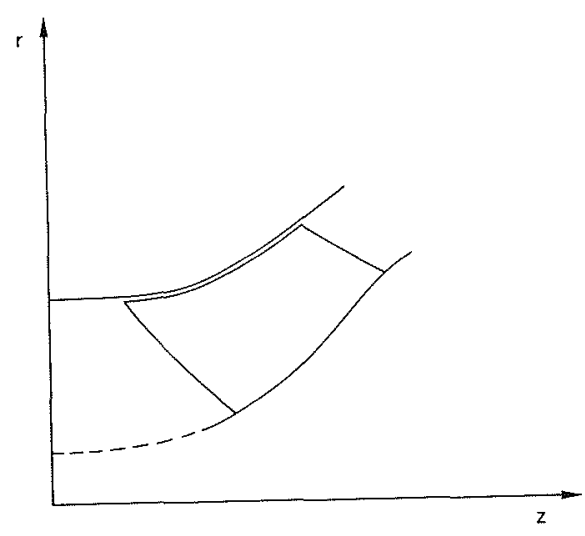

Figure 4 - Tracé đu canal méridien. 


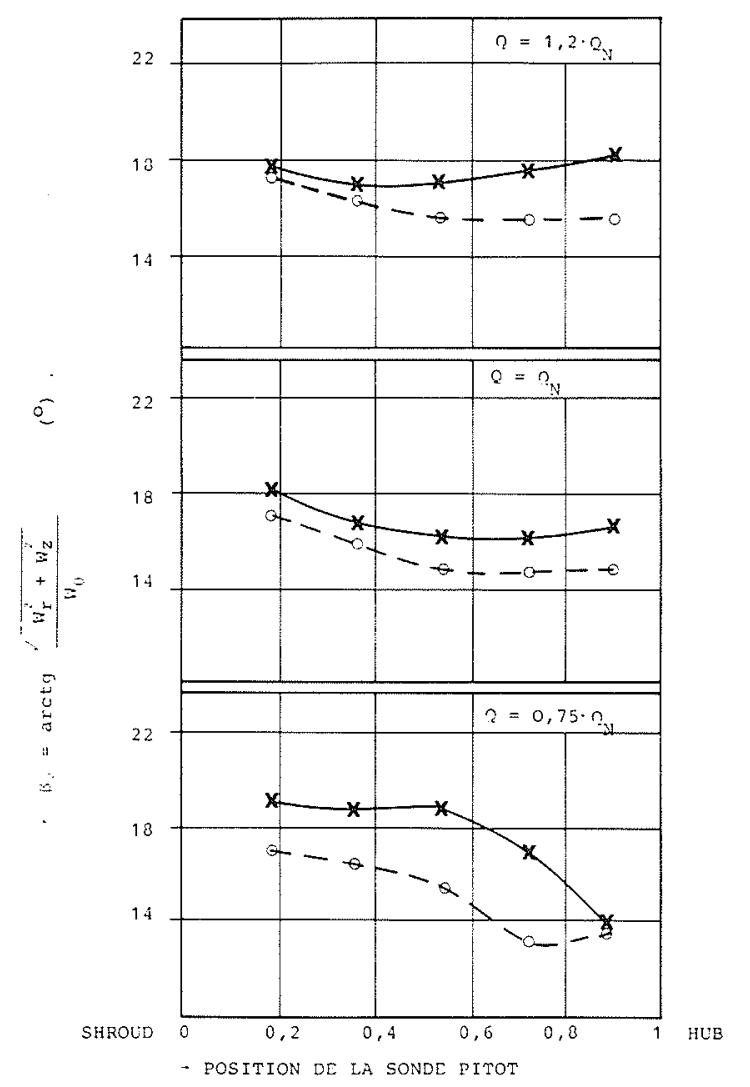

Figure 5 - Angles à la sortie de la roue $(\mathrm{X}=$ valeurs mesurées, $\mathrm{O}=$ valeurs calculées).

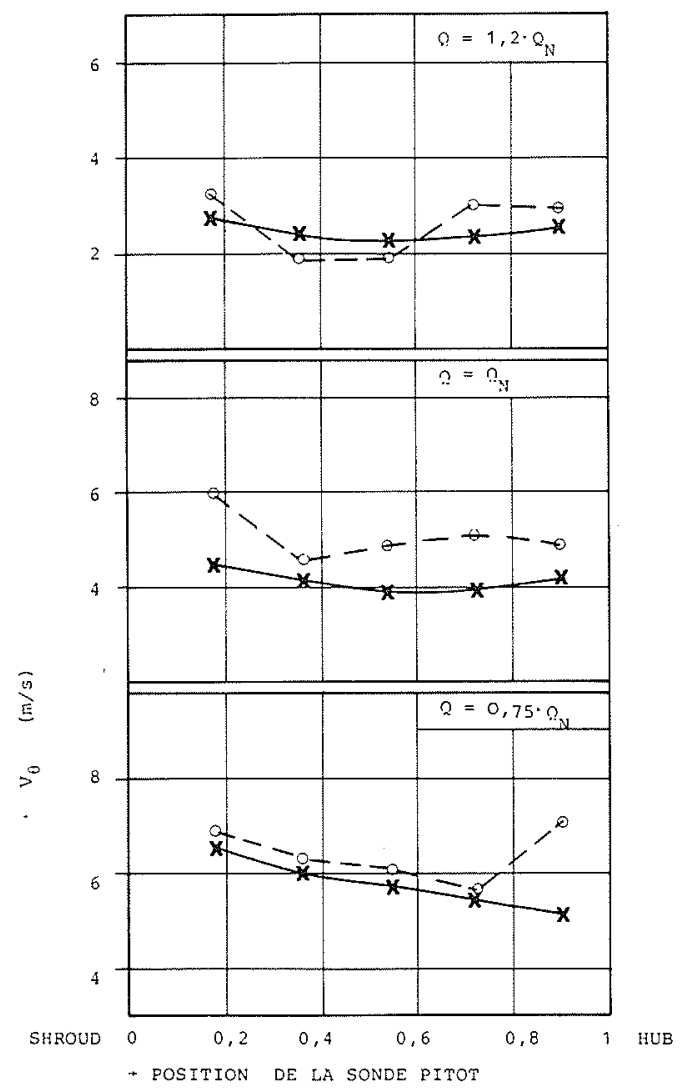

Figure 6 - Vitesse tangentielle à la sortie de la roue $(\mathrm{X}=$ valeurs mesurées, $\mathrm{O}=$ valeurs calculées)

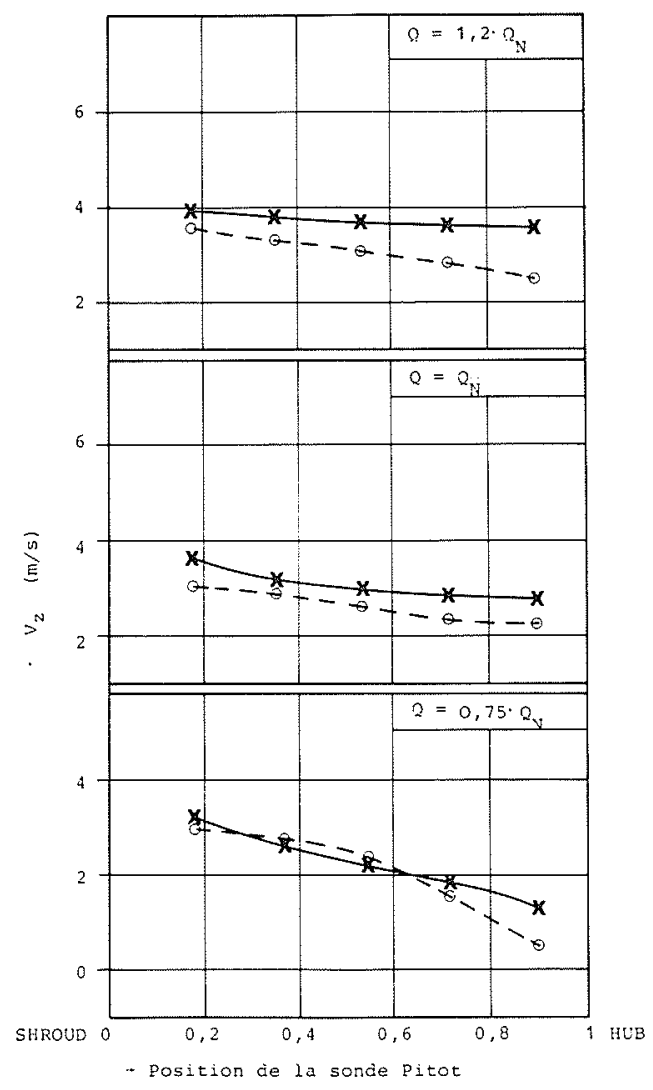

Figure 7 - Composante radiale de la vitesse à la sortie de la roue $(\mathrm{X}=$ valeurs mesurées, $\mathrm{O}=$ valeurs calculées $)$.

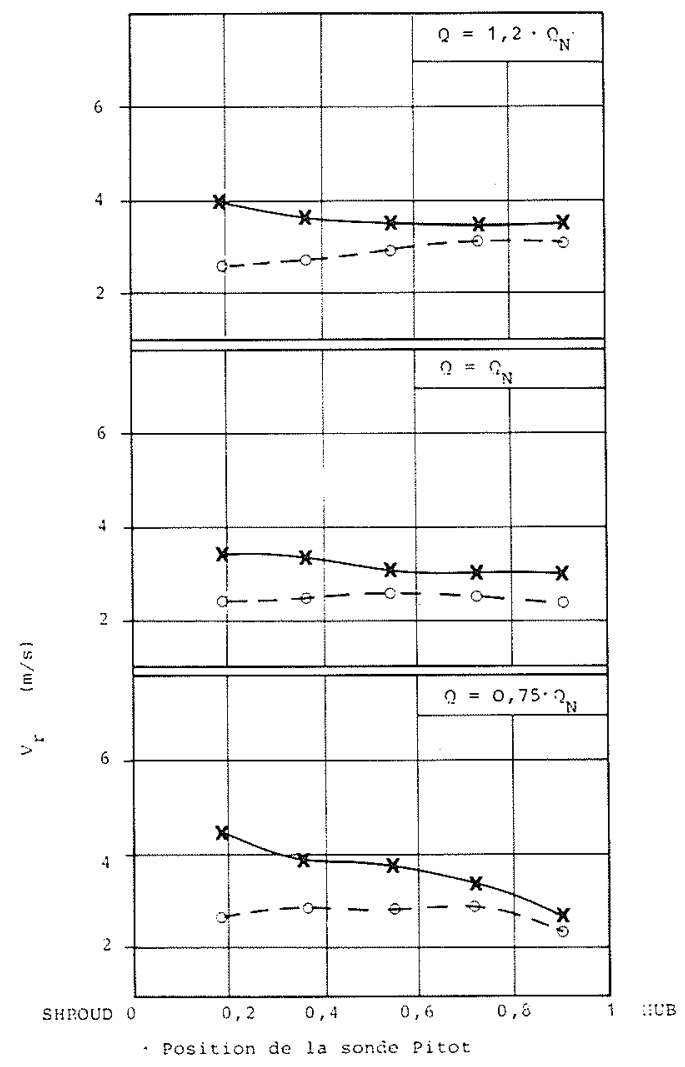

Figure 8 - Composante axiale de la vitesse à la sortie de la roue ( $\mathrm{X}=$ valeurs mesurées, $\mathrm{O}=$ valeurs calculées). 


\section{Références}

[1] BOSMAN C., MARSH H. - An Improved Method for Calculating Flow in Turbo-machines, Including a Consistent Loss Model. J. Mech Engineering Science, Vol. 16, $\mathrm{n}^{\circ} 1$, 1974.
[2] CARNEVALE E., GIUSTI S. - Analisi del flusso meridiano non isoentropico in giranti di pompe centrifughe con il metodo degli elementi finiti. XXXVI Congresso Nazionale ATI, Padova, 1982.

[3] WIESNER F.J. - A Review of Slip Factors for Centrifugal Impellers. J. of Eng. for Power - ASME - Oct. 1967.

\section{Discussion}

\section{Président : S. CASACCI}

Sur l'intervention de M. BORCIANI concernant l'introduction des pertes dans le calcul, M. ANGELO explique qu'elles ont été prises en compte sur la base de résultats expérimentaux acquis sur d'autres pompes de même type, la campagne d'essais sur cette pompe n'ayant pas encore commencé lors du calcul.

Sur la demande de M. VENTRONE, M. ANGELO précise que le débit considéré est le débit prévu pour la pompe.

En réponse à M. SAGNER s'étonnant de ce que les vitesses calculées soient systématiquement inférieures à celles des essais et demandant si le débit est le même dans les deux cas, M. ANGELO explique que les couches limites n'ont pas été prises en compte dans le calcul. La section de passage de l'écoulement dans la réalité étant donc inférieure à celle du calcul, les vitesses mesurées sont donc obligatoirement supérieures pour un même débit.

M. CASACCI ajoute que ce problème n'est pas appanu dans le cas présenté par M. EREMEEF en raison de l'absence de décollement sur les aubes dans une turbine. Il confirme que dans le cas d'une pompe, la mesure donnera des vitesses plus grande à cause de la présence de sillages alors que le calcul, ne tenant pas compte des décollements, donnera une répartition de vitesses plus uniforme et plus faible.

M. EREMEEF signale qu'il faut aussi tenir compte du blocage pariétal dans le cas d'une pompe.

M. CASACCI conclut que la divergence de la veine dans une pompe rend les calculs plus délicats qu'en turbine.

\section{Abstract \\ Theoretical - experimental analysis of real flows in assoradial pumps}

The paper presents a numerical method for non-isoentropic through flow calculation in centrifugal and mixed-flow pump impellers. The analysis of incompressible fluid motion is done following Wu's general theory on $S 2$ type surface. In particular, one of the motion equations is substituted by the geometrical condition for the flow to follow the prescribed $S 2$ surface.

Besides the flow is assumed to take place in presence of a dissipative force field locally opposite to the relative velocity vector and tangent to the stream line.

Combining the basic equations of continuity, entropy production, energy and motion, defining the local geometry of the stream surface $S 2$ (angles $\lambda$ and $\mu$ ) and the stream function $\psi$ it is possible to derive the final flow equation of the type

$$
\frac{\bar{\partial}^{2} \psi}{\partial r^{2}}+\frac{\bar{\partial}^{2} \psi}{\partial z^{2}}=q_{I}+q_{E}
$$

where $q_{I}$ depends on velocity components and on $S 2$ geometry and $q_{E}$ depends on the entropy production inside the fluid flow.

The equation of stream surface is obtained starting from the blade geometry and considering its distortion due to the slip effect. For the expression of entropy gradient a local loss coefficient $\eta(\psi)$ is defined as a ratio between the rise of total pressure in the real flow conditions and the rise of total pressure in isoentropic flow. In this way it is possible to evaluate the therm $q_{E}$ if a preceding isoentropic flow calculation is available.

The computer numerical model for the through flow calcuIation performs the following steps:

a) definition of $S 2$ geometry starting from the impeller geometric data and considering the slip effect.

b) Iterative solution of equation (7) in the isoentropic case $\left(q_{E}=0\right)$

c) Evaluation of entropy field.

d) Iterative solution of equation (7) in its complete form. In the step a) three-dimensional parametric splines are used for surface representation. The discretization of the problem is done using finite element technique with isoparametric, rectangular elements (8 nodes).

The solution of non-linear equation (7) is performed using Galerkin's weighted residual method.

The paper finally shows an example of through flow calculation in a mixed-flow pump for the design conditions and for two off-design working points. The computed results are compared with experimental measurements. 